\title{
Respiratory Motion Estimation from Cone-Beam Projections Using a Prior Model
}

\author{
Jef Vandemeulebroucke ${ }^{1,2,3}$, Jan Kybic ${ }^{3}$, Patrick Clarysse ${ }^{1}$, \\ and David Sarrut ${ }^{1,2}$ \\ 1 University of Lyon, CREATIS-LRMN; CNRS UMR5220; INSA-Lyon, France \\ 2 University of Lyon, Léon Bérard Cancer Center, F-69373, Lyon, France \\ 3 Center for Machine Perception, Czech Technical University in Prague, \\ Czech Republic
}

\begin{abstract}
Respiratory motion introduces uncertainties when planning and delivering radiotherapy for lung cancer patients. Cone-beam projections acquired in the treatment room could provide valuable information for building motion models, useful for gated treatment delivery or motion compensated reconstruction. We propose a method for estimating $3 \mathrm{D}+\mathrm{T}$ respiratory motion from the $2 \mathrm{D}+\mathrm{T}$ cone-beam projection sequence by including prior knowledge about the patient's breathing motion. Motion estimation is accomplished by maximizing the similarity of the projected view of a patient specific model to observed projections of the cone-beam sequence. This is done semi-globally, considering entire breathing cycles. Using realistic patient data, we show that the method is capable of good prediction of the internal patient motion from cone-beam data, even when confronted with interfractional changes in the breathing motion.
\end{abstract}

\section{Introduction}

In radiotherapy, breathing motion causes uncertainties in the dose delivered to the tumor. The existing approaches to take respiratory motion into account include adding safety margins to ensure target coverage, breath-hold, gating, or tracking of the target [1. An important prerequisite to plan and evaluate treatment when using these techniques is a detailed knowledge of the motion. Four-dimensional (4D) computed tomography imaging [2] or cone-beam (CB) CT [3], consisting of three dimensional (3D) frames each representing a breathing phase, can provide additional motion information. However, no intercycle variability can be measured as they represent a single respiratory cycle.

Breathing motion occurs predominantly in cranio-caudal direction and tends to be larger for the lower lobes [1. Trajectories of tumors and organs can be subject to hysteresis [4], i.e. a different path is followed during inhalation and exhalation. Cycles can differ from one another in breathing rate and level [5]; the latter influencing the amplitude of the motion. Variations in the mean tumor position (baseline) between and during fractions have also been reported [4]6].

Previously, 4D CT [7] and cine CT volume segments covering multiple cycles [8] have been used to model breathing motion. The small amount of acquired 
breathing cycles limits their ability to model intercycle variability. 4D MRI [9] covering more cycles could offer a solution to this problem. Regardless of the chosen approach, one should be able to detect and correct for interfractional changes in breathing motion that occur frequently between treatment sessions 46].

A CB projection sequence consists of a series of wide angle X-ray projections taken from viewpoints orbiting the patient. CBCT is routinely acquired for patient setup in many institutions, immediately before treatment, with the patient in the treatment position. Zijp et al. [10] have a fast and robust method for extracting a respiratory phase signal from a CB projection sequence. By establishing a relation to a prior 4D CT, Rit et al. [1] obtained a motion model that proved suitable for motion compensated CB reconstruction. Zeng et al. [12] estimated motion from a projection sequence by deforming a reference CT image so that its projection views match the $\mathrm{CB}$ sequence. Optimization of the large number of parameters of a B-spline based deformation model required adding aperiodicity penalties to the cost function to regularize the problem.

This article deals with in situ motion estimation from CB projection data for radiotherapy of lung cancer. With respect to [12] we incorporate prior knowledge in the form of a patient-specific model, significantly reducing the number of parameters to be identified. No intercycle regularization is required and we obtain improvement in speed and robustness. Within-cycle smoothness is guaranteed automatically, through the use of a B-spline temporal model.

\section{Method}

First, a parametric patient-specific motion model with a small number of degrees of freedom is built from a 4D CT image routinely acquired preoperatively for the irradiation treatment planning of the considered patient group. The model is able to represent changes in the breathing phase in addition to small variations in breathing pattern. The model is then fitted to the CB projection sequence by optimizing the model parameters to maximize the similarity between the acquired 2D CB projections and simulated projection views of the model. Individual cycles are processed separately and a smooth motion estimate is found by simultaneously considering the whole cycle with suitable boundary conditions.

\subsection{Motion Model}

Using the demons algorithm [13, we deformably register a manually chosen reference frame $f_{*}$ to all other frames $f_{\vartheta}$ of the $4 \mathrm{D} \mathrm{CT}$, where $\vartheta \in[0 ; 1)$ is the breathing phase. $f_{*}$ should be chosen as to contain as little artifacts as possible. End-exhale is usually a good choice. Let $g_{\vartheta}(\mathbf{x})$ be the resulting deformation vector field, mapping $f_{*}$ to $f_{\vartheta}$. All deformation fields are averaged and a mean position image $\bar{f}$ is created by backward warping of $f_{*}$ [14] (Figure 14).

$$
\bar{f}(\mathbf{x})=f_{*}\left(\bar{g}^{-1}(\mathbf{x})\right) \quad \text { with } \quad \bar{g}(\mathbf{x})=\frac{1}{b} \sum_{\theta=1}^{b} g_{\theta}(\mathbf{x})
$$




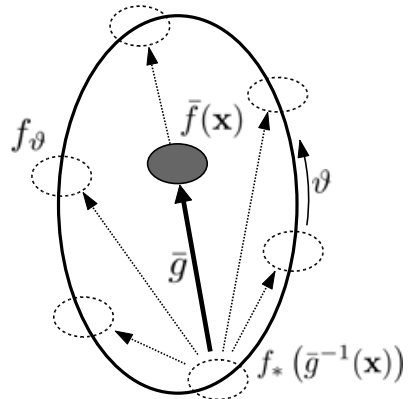

(a)

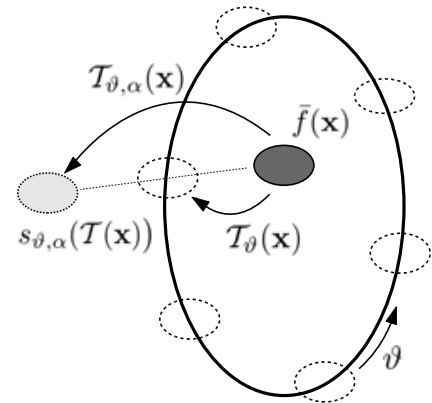

(b)

Fig. 1. (a) The procedure for obtaining a mean position image $\bar{f}$. (b) A schematic representation of the representable space for the proposed model. Consider a point with position $\mathbf{x}$ in in the image $\bar{f}$ (dark oval). Its position in all frames of the 4D CT (white ovals) is interpolated yielding the estimated breathing trajectory (bold curve). The amplitude parameter $\alpha$ allows to reach breathing states $s_{\vartheta, \alpha}$ off the trajectory.

All structures appear at their time-weighted mean position in the image $\bar{f}[15]$. Next, $\bar{f}$ is registered to the original frames $f_{\vartheta}$. The resulting deformation fields are approximated using B-splines as

$$
\mathcal{T}_{\vartheta, \alpha}(\mathbf{x})=\mathbf{x}+\alpha \sum_{\mathbf{i}} \sum_{j} a_{\mathbf{i} j} \beta^{n_{\mathbf{x}}}\left(\frac{\mathbf{x}-\mathbf{x}_{\mathbf{i}}}{\Delta_{\mathbf{x}}}\right) \beta^{n_{\vartheta}}\left(\frac{\vartheta-\vartheta_{j}}{\Delta_{\vartheta}}\right) .
$$

where $\beta^{n}($.$) are B-splines placed at positions \mathbf{x}_{\mathbf{i}}, \vartheta_{j}$ with uniform spacing $\Delta_{\mathbf{x}}$, $\Delta_{\vartheta} ; a_{\mathbf{i} j}$ are the B-spline coefficients. As $\vartheta$ varies from 0 to 1 , the deformation model produces a motion corresponding to an entire breathing cycle starting from end-exhalation. Note that this allows to model hysteresis. The second parameter $\alpha$ is an instantaneous amplitude (it can vary with $\vartheta$ ) and helps to model variations of the trajectory shape and breathing level. We chose cubic spline interpolation for phase space $\left(n_{\vartheta}=3\right)$. For the spatial dimension however, since dense deformation fields are available, a fast nearest neighbor $\left(n_{\mathbf{x}}=0\right)$ is employed. The coefficients $a_{\mathbf{i} j}$ are found quickly using digital filtering [16]. Image $s_{\vartheta, \alpha}$ for a particular breathing state described by $\vartheta, \alpha$ (Figure 10) is obtained through forward warping [14] (where the subscript for $\mathcal{T}$ was omitted)

$$
s_{\vartheta, \alpha}(\mathcal{T}(\mathbf{x}))=\bar{f}(\mathbf{x}) .
$$

\subsection{Cost Function and Optimization Strategy}

We propose to optimize the parameters of the model together for each breathing cycle. This renders the method more robust with respect to simply considering each projection separately (see Section 3), but is computationally more tractable than a truly global optimization (over many breathing cycles). Since breathing cycle extrema can usually be identified well, the accuracy is not compromised. 


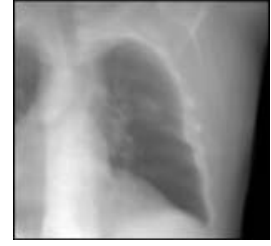

(a)

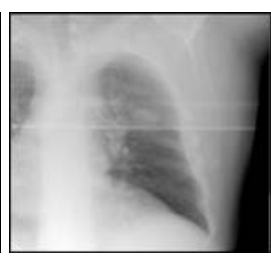

(b)

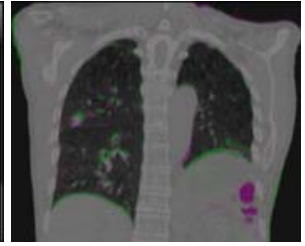

(c)

Fig. 2. (a) A simulated CB projection view calculated from the mean position image $\bar{f}$ and (b) a true CB projection of the same patient from the same viewpoint. Note that the images are very similar except for a horizontal reinforcement of the treatment table visible in the true $\mathrm{CB}$ projection. (c) Color overlay of preregistered end-inhalation frames from the two 4D CT acquisitions of patient 1 .

Given a CT volume $f$, an ideal cone-beam projection image $p$ can be calculated using a linear projection operator $\mathcal{P}_{\phi}$ where the parameter $\phi$ fully describes the (known) camera position and orientation:

$$
p=\mathcal{P}_{\phi} f
$$

Figure 2] and 2] show a CB projection view of a mean position image $\bar{f}$ compared with a $\mathrm{CB}$ projection of the same patient. We measure similarity between an observed $\mathrm{CB}$ projection $\hat{p}$ and a modeled breathing state $s_{\vartheta, \alpha}$ by calculating the normalized correlation coefficient (NCC) in the 2D projection space:

$$
J(\vartheta, \alpha ; \phi)=\operatorname{NCC}\left(\hat{p}, \mathcal{P}_{\phi} s_{\vartheta, \alpha}\right) .
$$

In a first step, we detect the approximate time positions (projection indexes) $t_{e}$ corresponding to extreme breathing phases [10. The method is based on taking image derivatives and analyzing 1D projections of the obtained image. Second, we refine the parameters $\vartheta\left(t_{e}\right)$ and $\alpha\left(t_{e}\right)$ by minimizing

$$
J\left(\vartheta\left(t_{e}\right), \alpha\left(t_{e}\right) ; \phi\right)+w\left(\vartheta\left(t_{e}\right)-\vartheta_{e}\right) \quad \text { with } \quad w(y)= \begin{cases}0 & \text { for }|y| \leq h \\ \delta|y|^{2} \text { otherwise } .\end{cases}
$$

Note we are favoring solutions near the expected phase value $\vartheta_{e}$. Powell-Brent 17 multidimensional search was used with $h=0.1$ and $\delta=20$ with initial values $\alpha\left(t_{e}\right)=1$ and $\vartheta\left(t_{e}\right)=\vartheta_{e}=\vartheta_{e e}$ or $\vartheta_{e i}$ for end-exhalation and end-inhalation, respectively. The values for both $\vartheta_{e e}$ and $\vartheta_{e i}$ were determined by applying the extrema detection method [10] to simulated projections of the model with slowly varying phase.

Let $t_{e}$ and $t_{e^{\prime}}$ be the two end-inhalation positions, the beginning and end of a breathing cycle. We have just shown how to get $\vartheta$ and $\alpha$ at $t_{e}, t_{e^{\prime}}$, what remains is to obtain the estimates also for frames $t_{e+1}, \ldots, t_{e^{\prime}-1}$. Assuming temporal smoothness, we propose to represent $\vartheta$ as

$$
\vartheta(t)=\sum_{i=0}^{k} c_{i} \beta^{n_{\vartheta_{t}}}\left(\frac{t-t_{i}}{\Delta_{\vartheta_{t}}}\right) \text { for } t_{e}<t<t_{e^{\prime}}
$$


where $k$ is the number of control points, $t_{i}$ are the temporal position of the knots, $\Delta_{\vartheta_{t}}$ is the knot spacing and $c_{i}$ are the B-spline coefficients. Fixing the value for $\vartheta\left(t_{e}\right)$ we can express the boundary coefficient $c_{0}$ as

$$
c_{0}=\frac{\vartheta\left(t_{e}\right)-\sum_{i=1}^{k} c_{i} \beta^{n_{\vartheta_{t}}}\left(\frac{t_{e}-t_{i}}{\Delta_{\vartheta_{t}}}\right)}{\beta^{n_{\vartheta_{t}}}\left(\frac{t_{e}-t_{0}}{\Delta_{\vartheta_{t}}}\right)} .
$$

and similarly for $c_{k}$. A B-spline expansion with coefficients $d_{j}$ is used to represent $\alpha(t)$. By summing the contributions for $m$ different time instances within the cycle and using equations (5), (7) 8 , we obtain the following similarity measure:

$$
J^{t}(\mathbf{c}, \mathbf{d})=\frac{1}{m} \sum_{t=1}^{m} J\left(\vartheta\left(t_{e}+t\right), \alpha\left(t_{e}+t\right) ; \phi\left(t_{e}+t\right)\right) .
$$

We find the coefficients $\mathbf{c}=\left[c_{1}, \ldots, c_{k}\right], \mathbf{d}=\left[d_{1}, \ldots, d_{l}\right]$ by minimizing $J^{t}$, using a Nelder-Nead downhill simplex algorithm [17, which performed well in this high dimensional search space, requiring less iterations than Powell-Brent and yielding comparable results. A linear progression is used as a starting point. We use a quadratic B-spline representation $\left(n_{\vartheta_{t}}=n_{\alpha_{t}}=2\right)$ with $k=l=4$.

\section{Experiments and Results}

Accurately evaluating the 2D-3D motion estimation is very difficult, as no ground truth is available. In this work we use pairs of $4 \mathrm{D} \mathrm{CT}$ sequences acquired for three lung cancer patients using a Philips Brilliance BigBore 16-slice CT scanner (Philips Medical Systems, Cleveland, OH). The time between acquisitions ranged from 20 minutes (patient 1 and 2) to 3 days (patient 3). Patients 1 and 2 were asked to stand up from the acquisition table and walk around for 10

(a)
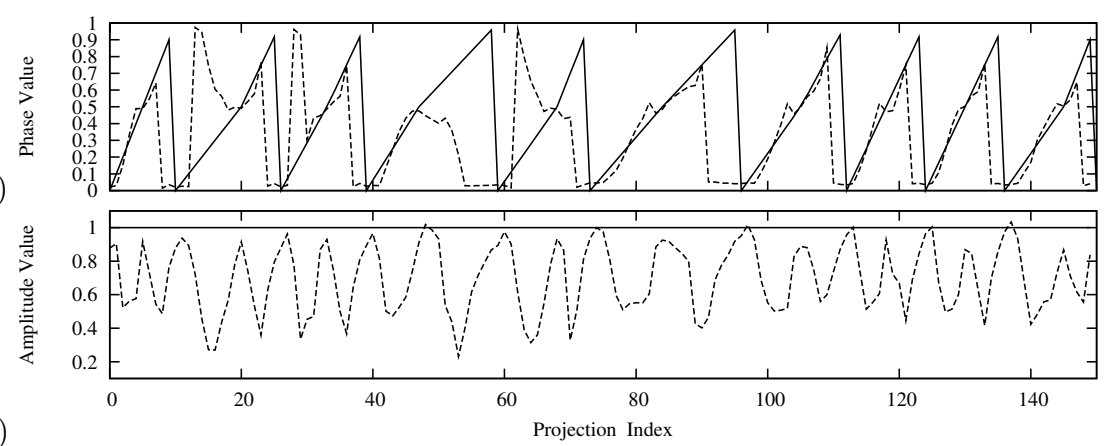

Fig. 3. Results of sequential motion estimation for patient 1: the recovered phase (a) and amplitude (b) (dashed line) together with the parameters used to generate the $\mathrm{CB}$ sequence (full line). The reference amplitude is a constant, $\alpha=1$. 
Table 1. Results of the semi-global motion estimation. The residual misalignment (residual) between the found and the true motion: the mean $(\mu)$, standard deviation $(\sigma)$ and maximum $(\max )$ is compared to the original motion with respect to $\bar{f}$ (original).

\begin{tabular}{c||ccc|ccc}
$(\mathrm{mm})$ & \multicolumn{3}{||}{ original } & \multicolumn{3}{c}{ residual } \\
\hline \hline & $\mu$ & $\sigma$ & $\max$ & $\mu$ & $\sigma$ & $\mathrm{Max}$ \\
\hline Patient 1 & 3.8 & 2.1 & 17.1 & 1.1 & 0.6 & 8.3 \\
Patient 2 & 2.8 & 1.7 & 16.1 & 1.6 & 0.8 & 11.0 \\
Patient 3 & 3.7 & 1.6 & 13.8 & 1.3 & 0.7 & 5.8
\end{tabular}

(a)

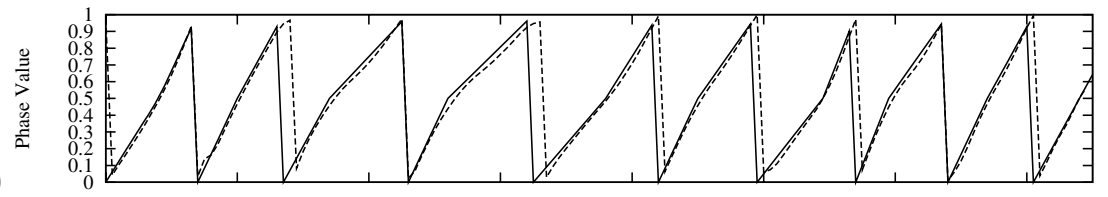

(b)

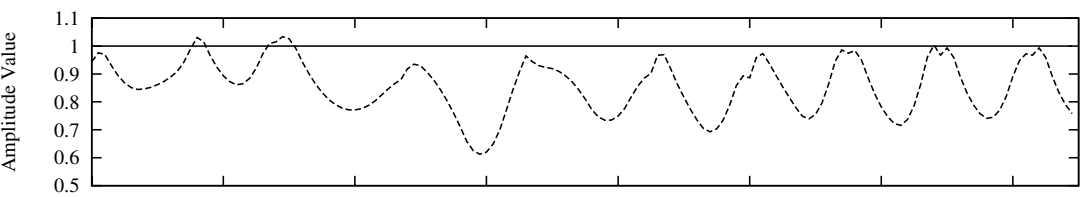

(c)

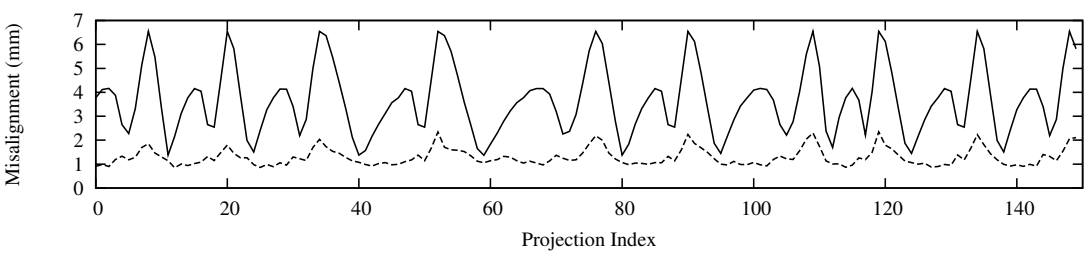

Fig. 4. Results of the semi-global motion estimation for Patient 3: the recovered phase (a) and amplitude (b) (dashed line) are shown together with the parameters used to generate the $\mathrm{CB}$ sequence (full line). The reference amplitude is a constant, $\alpha=1$. (c) The resulting residual misalignment (dashed line) is shown in comparison with the original misalignment with respect to the mean position image (full line).

minutes before repositioning and acquisition of the second 4D CT. In spite of the small time between the acquisitions, substantial difference can be observed between the two subsequent 4D CT acquisitions due to interfractional changes in breathing motion (see Figure 2r). We used the first 4D CT sequence to construct a patient model as described in Section 2.1. The second acquisition was first rigidly registered to the first 4D CT to align the bony structures. In order to have a ground truth available, we took the mean position image from the first sequence and the deformation fields from the second sequence to get a simulated reference 4D CT sequence. A respiratory trace was randomly generated [5] and a piece-wise linear phase signal $\vartheta(t)$ with variable breathing rate was derived. We simulated the first $90^{\circ}$ of a $\mathrm{CB}$ acquisition protocol in our institute by calculating 150 projections for evenly spaced angles from the reference 4D CT with varying phase value over a period of $30 \mathrm{~s}$. 
When optimizing separately for each projection the criterion (5) with respect to $\vartheta$ and $\alpha$, we obtained bad results when confronted to interfractional changes in breathing motion (see Figure 3, results for other patients were similar). Note that an optimal result doesn't necessarily mean recovering identical parameter values as they correspond to different deformation fields. In this case however, we can observe how intermediate phases during inhalation $(\vartheta \approx 0.2)$ and exhalation $(\vartheta \approx 0.8)$ are confused, due to limited hysteresis and unfavorable projection angle and are accompanied with strong variations in amplitude.

The phase and amplitude found for Patient 3 using our semi-global criterion (9) are shown in Figure 4a and 4b, together with the parameters used to generate the CB sequence. To evaluate the accuracy we calculate the residual geometric misalignment (i.e. the norm of the difference between deformation vector fields) between the estimated motion and the true motion. This measure is averaged over the lower lung, where the largest motion tends to occur. For comparison, the original misalignment, i.e. the motion with respect to the mean position image, is also given. Table 1 contains the average over all projections for each patient. Figure 4 shows this mean misalignment as a function of the projection index for Patient 3. Note that while displacement might locally attain $3 \mathrm{~cm}$, the average motion as seen from the mean position does not exceed $1 \mathrm{~cm}$.

\section{Discussion and Conclusion}

We achieved a smooth motion estimation from a CB projection sequence using B-splines and by considering the complete movement in a respiratory cycle and obtained a considerable reduction of the original misalignment for all patients.

To our knowledge this is the first time a respiratory motion model is tested against clinical data containing real interfractional changes in breathing motion. Some additional challenges presented by real CB data will include dealing with scatter 12 and detecting and correcting for rigid misalignment (setup errors).

As a consequence of generating the ground truth, baseline shifts were not present in our patient data. Changes in breath rate, breathing level or trajectory shape were however present. It is expected that the method will be able to cope with small shifts ( $<20 \%$ of the motion amplitude). For larger shifts, a prior shift estimation can be performed, e.g. from a 4D CBCT [6].

In this work we exploited only acquisitions already acquired for treatment purposes. More preoperative data, such as breath hold CT scans [812] or MRI data [9], could further improve the prior model, rendering it's construction more robust to artifacts and providing prior information on the intercycle variation.

Acknowledgments. Jef Vandemeulebroucke was funded by the EC Marie Curie grant WARTHE, Jan Kybic was sponsored by the Czech Ministry of Education, Project MSM6840770012. 


\section{References}

1. Keall, P.J., Mageras, G.S., Balter, J.M., Emery, R.S., Forster, K.M., Jiang, S.B., Kapatoes, J.M., Low, D.A., Murphy, M.J., Murray, B.R., Ramsey, C.R., Herk, M.B.V., Vedam, S.S., Wong, J.W., Yorke, E.: The management of respiratory motion in radiation oncology report of AAPM task group 76. Med. Phys. 33(10), 3874-3900 (2006)

2. Ford, E.C., Mageras, G.S., Yorke, E., Ling, C.C.: Respiration-correlated spiral $\mathrm{CT}$ : a method of measuring respiratory-induced anatomic motion for radiation treatment planning. Med. Phys. 30(1), 88-97 (2003)

3. Sonke, J., Zijp, L., Remeijer, P., van Herk, M.: Respiratory correlated cone beam CT. Med. Phys. 32(4), 1176-1186 (2005)

4. Seppenwoolde, Y., Shirato, H., Kitamura, K., Shimizu, S., van Herk, M., Lebesque, J.V., Miyasaka, K.: Precise and real-time measurement of 3D tumor motion in lung due to breathing and heartbeat, measured during radiotherapy. Int. J. Radiat. Oncol. Biol. Phys. 53(4), 822-834 (2002)

5. George, R., Vedam, S.S., Chung, T.D., Ramakrishnan, V., Keall, P.J.: The application of the sinusoidal model to lung cancer patient respiratory motion. Med. Phys. 32(9), 2850-2861 (2005)

6. Sonke, J.J., Lebesque, J., van Herk, M.: Variability of four-dimensional computed tomography patient models. Int. J. Radiat. Oncol. Biol. Phys. 70(2), 590-598 (2008)

7. Zhang, Q., Pevsner, A., Hertanto, A., Hu, Y.C., Rosenzweig, K.E., Ling, C.C., Mageras, G.S.: A patient-specific respiratory model of anatomical motion for radiation treatment planning. Med. Phys. 34(12), 4772-4781 (2007)

8. McClelland, J.R., Blackall, J.M., Tarte, S., Chandler, A.C., Hughes, S., Ahmad, S., Landau, D.B., Hawkes, D.J.: A continuous 4D motion model from multiple respiratory cycles for use in lung radiotherapy. Med. Phys. 33(9), 3348-3358 (2006)

9. von Siebenthal, M., Székely, G., Gamper, U., Boesiger, P., Lomax, A., Cattin, P.: 4D MR imaging of respiratory organ motion and its variability. Phys. Med. Biol. 52(6), 1547-1564 (2007)

10. Zijp, L., Sonke, J., van Herk, M.: Extraction of the respiratory signal from sequential thorax cone-beam X-ray images. In: 14th International Conference on the Use of Computers in Radiation Therapy, Seoul, Korea (May 2004)

11. Rit, S., Wolthaus, J., van Herk, M., Sonke, J.-J.: On-the-fly motion-compensated cone-beam CT using an a priori motion model. In: Metaxas, D., Axel, L., Fichtinger, G., Székely, G. (eds.) MICCAI 2008, Part I. LNCS, vol. 5241, pp. 729-736. Springer, Heidelberg (2008)

12. Zeng, R., Fessler, J.A., Balter, J.M.: Estimating 3-D respiratory motion from orbiting views by tomographic image registration. IEEE Trans. Med. Imaging 26(2), 153-163 (2007)

13. Thirion, J.P.: Image matching as a diffusion process: an analogy with Maxwell's Demons. Med. Image Anal. 2(3), 243-260 (1998)

14. Wolberg, G.: Digital image warping. IEEE Computer Society Press, Los Alamitos (1990)

15. Wolthaus, J.W.H., Sonke, J.J., van Herk, M., Damen, E.M.F.: Reconstruction of a time-averaged midposition CT scan for radiotherapy planning of lung cancer patients using deformable registration. Med. Phys. 35(9), 3998-4011 (2008)

16. Unser, M.: Splines: A perfect fit for signal and image processing. IEEE Signal Processing Magazine 16(6), 22-38 (1999)

17. Press, W.H., Flannery, B.P., Teukolsky, S.A., Vetterling, W.T.: Numerical Recipes in C, 2nd edn. Cambridge University Press, Cambridge (1992) 